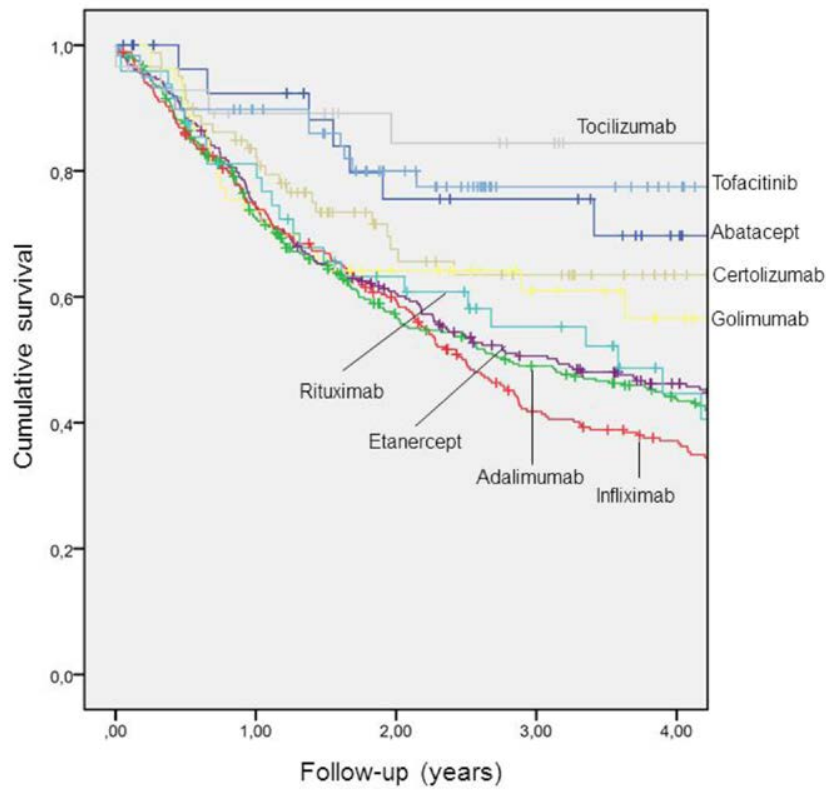

Conclusion: In our study, infliximab was related to an overall higher hazard of treatment course interruption than tolicizumab and tofacitinib, and higher hazard of interruption due to AEs than most other anti-TNF agents and tofacitinib. Maintaining antimalarials in patients receiving advanced therapies for RA may reduce interruption of treatment due to inefficacy and AEs.

Disclosure of Interests: None declared

DOI: 10.1136/annrheumdis-2021-eular.3841

\section{POS0677 THE ROLE OF MUSCULOSKELETAL ULTRASOUND IN PREDICTING THE RESPONSE TO JAK INHIBITORS: RESULTS FROM A LARGE MONOCENTRIC COHORT}

C. Garufi ${ }^{1}$, F. Ceccarelli ${ }^{1}$, F. R. Spinelli ${ }^{1}$, S. Mancuso ${ }^{1}$, C. Pirone ${ }^{1}$, F. Conti ${ }^{1}$. ${ }^{1}$ Sapienza University of Rome, Reumatologia, Dipartimento di Dipartimento di Scienze Cliniche Internistiche, Anestesiologiche e Cardiovascolari, Roma, Italy

Background: In the management of chronic arthritis, such as Rheumatoid Arthritis (RA), Ultrasound (US) assessment can provide relevant information about the joint inflammatory status in the diagnostic phase and even more in the monitoring of disease activity and structural damage ${ }^{1,2}$.

Objectives: In this longitudinal study, we aimed to assesse the role of US in predicting the efficacy of JAK-inhibitors (JAKi) in RA patients.

Methods: We enrolled RA patients starting baricitinib or tofacitinib. All patients were evaluated at baseline and after 4, 12, 24, 48 weeks. Disease activity was calculated by DAS28CRP. US examination in 22 joints (I-V MCPs and PIPs, wrists) aimed at evaluating inflammatory features (synovial effusion and hypertrophy, power Doppler-PD), through a semi-quantitative scale (0-3). The total US $(0-198)$ and PD (0-66) scores were calculated. We scanned bilateral flexor (I-V fingers of hands) and extensor compartments (1-6) tendons: tenosynovitis was scored as absent/present (0/1), resulting in a total score (0-22).

Results: We studied 102 patients (M/F 15/87; median age 59.2 years, IQR 17.75; median disease duration 144 months, IQR 126), 61 treated with baricitinib and 41 with tofacitinib. At baseline, the median total US score was 18 (IQR 19) and the median PD score 2 (4). We observed a significant reduction in both total and PD US scores at all time-points $(p<0.0001$ ) (Figure 1). At baseline, $75.4 \%$ of patients showed tenosynovitis involving at least one tendon, with a median score of 2 (IQR 3.5) significantly decreasing after 24 weeks $(p=0.02)$. Multivariate analysis, adjusted for baseline DAS28 ${ }_{\text {CRP }}$ and other concomitant treatments (including glucocorticoids and methotrexate treatment), confirmed the independent association between baseline US (PD and tenosynovitis) scores and the reduction of disease activity at follow-up evaluations.

Conclusion: The present study confirmed the early efficacy of JAKi in RA patients by using US evaluation. Furthermore, power doppler and tenosynovitis scores could play a predictive role in response to treatment.

REFERENCES:

[1] MUELLER RB, HASLER C, POPP F, et al. Effectiveness, Tolerability, and Safety of Tofacitinib in Rheumatoid Arthritis: A Retrospective Analysis of Real-World Data from the St. Gallen and Aarau Cohorts. J Clin Med. 2019;8(10):1548.

[2] COLEBATCH AN, EDWARDS CJ, ØSTERGAARD M, et al. EULAR recommendations for the use of imaging of the joints in the clinical management of rheumatoid arthritis. Ann Rheum Dis. 2013;72(6):804-14.

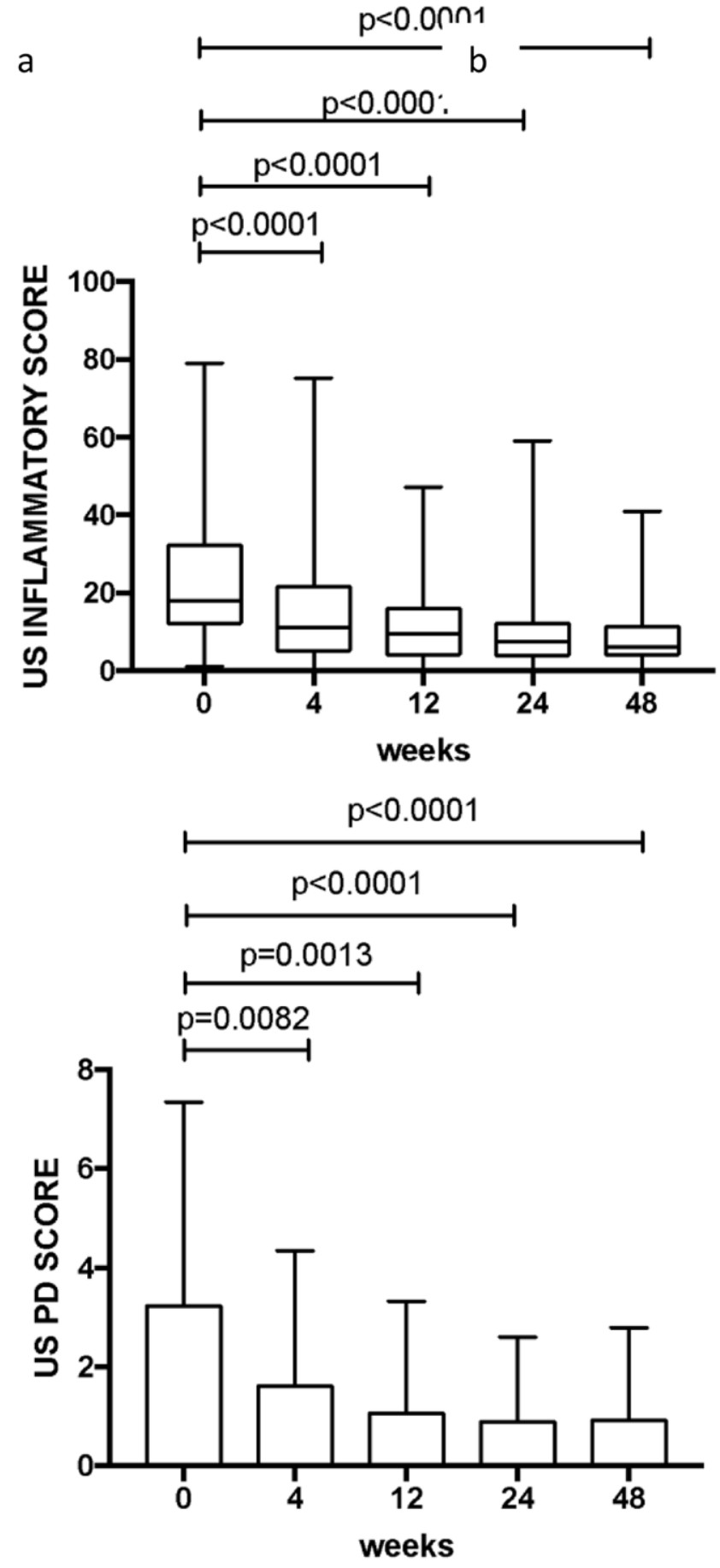

Figure 1. Ultrasound inflammatory score (a) and Ultrasound Power Doppler (PD) score (b) at baseline and follow-up.

\begin{tabular}{llllll}
\hline WEEKS & $\mathbf{0}$ & $\mathbf{4}$ & $\mathbf{1 2}$ & $\mathbf{2 4}$ & $\mathbf{4 8}$ \\
\hline US inflammatory score & $18(19)$ & $\begin{array}{l}11(15.5) \\
\text { US PD score }\end{array}$ & $\begin{array}{l}9.5(11.7) \\
0(1)\end{array}$ & $\begin{array}{l}7.5(8) \\
0(1)\end{array}$ & $\begin{array}{l}6(11) \\
0(0.7)\end{array}$ \\
\hline
\end{tabular}

Disclosure of Interests: Cristina Garufi: None declared, Fulvia Ceccarelli: None declared, Francesca Romana Spinelli Speakers bureau: Abbvie, Eli Lilly, Consultant of: Gilead/Galapagos, Eli Lilly, Grant/research support from: Pfizer, Silvia Mancuso: None declared, Carmelo Pirone: None declared, Fabrizio Conti Speakers bureau: Abbvie, Eli Lilly, Sanofi, Pfizer, Consultant of: Gilead/ Galapagos

DOI: 10.1136/annrheumdis-2021-eular.3850 